\title{
Distinct neural mechanisms mediate olfactory memory formation at different timescales
}

\author{
Ann Marie McNamara, ${ }^{1}$ Phillip D. Magidson, ${ }^{1}$ Christiane Linster, ${ }^{1}$ Donald A. Wilson, ${ }^{2}$ \\ and Thomas A. Cleland ${ }^{3,4}$ \\ ${ }^{1}$ Department of Neurobiology and Behavior, Cornell University, Ithaca, New York 14853, USA; ${ }^{2}$ Department of Zoology, \\ University of Oklahoma, Norman, Oklahoma 73019, USA; ${ }^{3}$ Department of Psychology, Cornell University, \\ Ithaca, New York 14853, USA
}

\begin{abstract}
Habituation is one of the oldest forms of learning, broadly expressed across sensory systems and taxa. Here, we demonstrate that olfactory habituation induced at different timescales (comprising different odor exposure and intertrial interval durations) is mediated by different neural mechanisms. First, the persistence of habituation memory is greater when mice are habituated on longer timescales. Second, the specificity of the memory (degree of cross-habituation to similar stimuli) also depends on induction timescale. Third, we demonstrate a pharmacological double dissociation between the glutamatergic mechanisms underlying short- and long-timescale odor habituation. LY341495, a class II/III metabotropic glutamate receptor antagonist, blocked habituation only when the induction timescale was short. Conversely, MK-801, an N-methyl-D-aspartate (NMDA) receptor antagonist, prevented habituation only when the timescale was long. Finally, whereas short-timescale odor habituation is mediated within the anterior piriform cortex, infusion of $\mathrm{MK}-801$ into the olfactory bulbs prevented odor habituation only at longer timescales. Thus, we demonstrate two neural mechanisms underlying simple olfactory learning, distinguished by their persistence and specificity, mediated by different olfactory structures and pharmacological effectors, and differentially utilized based solely on the timescale of odor presentation.
\end{abstract}

Habituation, or the progressive reduction in behavioral responsiveness to repeated similar stimuli, is a simple form of learning that underlies animals' capacity to perceptually deemphasize static conditions or inconsequential stimuli in favor of novel, potentially more relevant environmental features or events (Thompson and Spencer 1966; Rose and Rankin 2001). In the laboratory, experimental paradigms based on habituation have been employed to study the properties of nonassociative perceptual learning, to measure the persistence of memory traces, and even to assess the stringency of spontaneous discrimination among similar stimuli. In studies of olfactory spontaneous discrimination using rats or mice, animals habituated to an odorant stimulus demonstrate a cross-habituation response to novel odorant stimuli that are structurally and perceptually similar to the habituated odorant. The degree of cross-habituation declines progressively as test odorants become more dissimilar, and habituation to both the original and cross-habituated odorant stimuli persists for at least several minutes (Cleland et al. 2002; Linster et al. 2002; Mandairon et al. 2006b,c).

Olfactory habituation has also been observed in studies in which it is attributed to rapid synaptic adaptation of the mitrapyramidal synapse in the rat anterior piriform cortex (aPC) (Best and Wilson 2004; Yadon and Wilson 2005). Odor-evoked activity in piriform pyramidal neurons adapts within tens of seconds, and this adaptation persists for up to 2 min (Wilson 1998a,b), a substantially faster timescale than that exhibited in the behavioral studies discussed above. Furthermore, this activitydependent synaptic depression in the aPC is very specific to the habituation odor (Wilson 2000b), whereas adaptation of responses based on olfactory bulb mechanisms exhibits a broader cross-adaptation to similar odors (Wilson 2000a).

${ }^{4}$ Corresponding author.

E-mail tac29@cornell.edu; fax (775) 254-2756.

Article is online at http://www.learnmem.org/cgi/doi/10.1101//m.785608.
In the present study, we sought to determine the basis for the differences between these two forms of olfactory habituation. Specifically, we first asked whether the different timescales of odor presentation used could explain the apparent differences in the persistence and specificity of habituation suggested by these behavioral and physiological data. We subsequently sought to differentiate the two forms of habituation pharmacologically. In summary, our results demonstrate two fundamentally different types of olfactory habituation memory that involve two different glutamatergic systems in two different olfactory structures, encode memories of different duration and specificity, and operate at different timescales.

\section{Results}

\section{Persistence of habituation memory}

We first tested whether there were differences in memory persistence for a conspecific odor following habituation induced over either long or short timescales. Twenty-four mice were presented repeatedly with soiled bedding taken from an isolated conspecific male using either a short- (20-sec trials with 10 -sec intertrial intervals [ITIs]) or long- (50-sec trials with 5-min ITIs) timescale paradigm (Fig. 1; see Materials and Methods), and the time that they investigated the odor source during each trial was recorded. The habituated mice were then again presented with the same odor at a latency of either 10,20,30, or $60 \mathrm{~min}$ after the last habituation trial to measure how long the habituation memory persisted (Fig. 2A). Analysis of variance revealed a general effect of habituation timescale $\left(F_{\text {timescale }(1,368)}=98.18 ; P<0.001\right)$ and trial number $\left(F_{\text {trial }(11,368)}=27.09 ; P<0.001\right)$ on odor investigation time, as well as a significant interaction between the two $\left(F_{\text {timescale }} \times \operatorname{trial}(3,368)=19.6 ; P<0.001\right)$, indicating that the progression of habituation was significantly affected by the timescale of the habituation paradigm. 
A

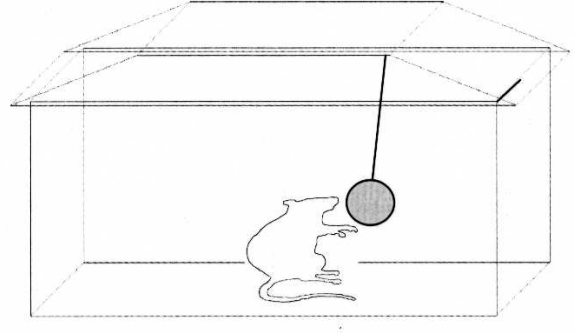

B

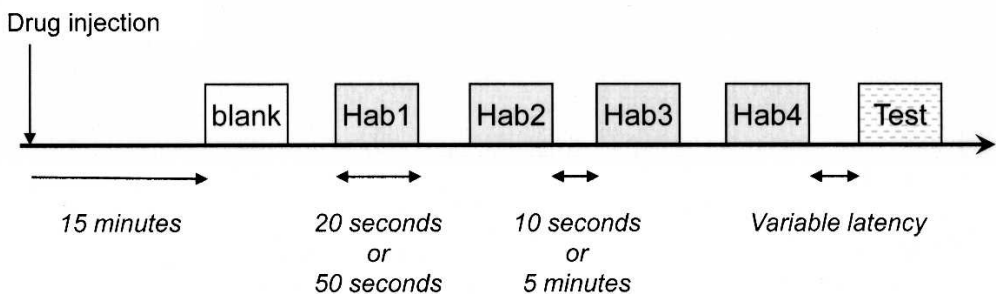

Figure 1. Experimental design. (A) Mice were separated into individual plastic cages with microisolator tops. For each trial, the cage was opened and a tea ball containing an odor source was hung within the cage so that the mouse could easily investigate it. The disturbance of placing the tea ball into the cage initiated each trial. (B) Habituation paradigms consisted of six trials: one blank trial in which clean bedding or plain mineral oil was presented to prehabituate mice to the non-olfactory features of stimulus presentation (these data are omitted from data figures and statistical analyses), four habituation trials in which soiled bedding or diluted acetic acid were presented as odorants, and, in some experiments, one test trial in which the habituation odorant or a structurally similar odorant was presented. In pharmacological experiments, the blank trial always began 15 min following drug injection. Two timescales of odorant presentation were used: either short (20-sec presentations with 10 -sec ITIs) or long (50-sec presentations with 5-min ITIs). For memory persistence experiments, the ITI between the last habituation trial and the test trial (latency) was the variable of interest and hence an exception to this rule. Soiled bedding from an isolated male conspecific was used as both the habituation and test odor except in memory specificity experiments, in which the habituation odorant was acetic acid and the test odorant was one of four structurally and perceptually similar odorants (propanoic, butanoic, pentanoic, or hexanoic acid).

The mice habituated using the short-timescale paradigm showed significant habituation to the conspecific odor (post hoc comparison of the first and fourth habituation trials; Tukey's honestly significant difference [HSD], $P<0.001)$. However, after only $10 \mathrm{~min}$ (the shortest test latency), the mice had recovered significantly from habituation; that is, they responded significantly more to the odor during the test trial than they had during the last habituation trial (Tukey HSD, $P<0.001$ ), indicating that the habituation memory persisted for $<10 \mathrm{~min}$ (Fig. 2A).

In the long-timescale paradigm, investigation times also habituated over the course of the four habituation trials (Tukey HSD, comparison between first and fourth trials, $P<0.001$; Fig. $2 \mathrm{~A})$. However, these mice remained habituated for $>30 \mathrm{~min}$ after the final habituation trial. Specifically, when tested 10,20 , or 30 min after the last habituation trial, their responses did not significantly differ from those measured during the last habituation trial (Tukey HSD, $P>0.05$ ). However, when tested at a 60 -min latency, the mice responded significantly more to the odor (Tukey HSD, $P=0.001$ ).

In order to more precisely determine the persistence of habituation memory following the short-timescale paradigm, a separate cohort of seven mice was trained on the short-timescale paradigm as described above; groups were then tested at latencies of $1,2,3,6$, or $8 \mathrm{~min}$ following the fourth habituation trial. As before, there was a significant effect of trial number $\left(F_{\text {trial }(4,182)}=20.78, P<0.001\right)$, and the mice were significantly habituated on the fourth trial (Tukey HSD, $P<0.001$ ). The results indicate that the habituation memory persisted for $>2$ min (Tukey HSD, comparison between fourth habituation trial and test trial; $P>0.05$ ) but $<3$ min (Tukey HSD, $P<0.005$; Fig. 2 B).
Spontaneous recovery from habituation after 2-3 min is in concordance with the behavioral and electrophysiological results attributed to homosynaptic depression in the aPC owing to habituation protocols performed at this timescale (Wilson 1998a,b; Best and Wilson 2004). Collectively, these results demonstrate that the timescale of olfactory habituation affects the persistence of the habituation memory.

\section{Specificity of habituation memory}

In short-timescale electrophysiological studies, aPC synaptic depression is highly specific, and rat piriform cortical neurons exhibit minimal crossadaptation between structurally and perceptually similar odorants (Wilson 2000b). Comparable results were observed behaviorally in a short-timescale habituation paradigm, in which little or no cross-habituation of similar odorants was observed (Fletcher and Wilson 2002). In contrast, rodents crosshabituate significantly to similar odorants in behavioral studies using a longertimescale habituation paradigm (Cleland et al. 2002). We employed a homologous series of sequentially similar carboxylic acid odorants to measure the specificity of cross-habituation to structurally similar odorants as a function of the timescale of odor presentation (Table 1). In a homologous series of unbranched aliphatic odorants, odorants with similar carbon chain lengths are correspondingly perceptually similar (Cleland et al. 2002). Analysis of variance revealed a general effect of habituation timescale $\left(F_{\text {timescale(1,485) }}=267.54, P<0.001\right)$ and trial number $\left(F_{\text {trial }(4,485)}=70.30, P<0.001\right)$, as well as a significant interaction between these factors $\left(F_{\text {trial }} \times\right.$ timescale $\left.(4,485)=12.63, P<0.001\right)$, indicating that the breadth of cross-habituation across a range of similar odorants was affected by the timescale of habituation.

Mice demonstrated significant habituation in both the short- and long-timescale paradigms comparable to that observed in response to conspecific odors (Tukey HSD, comparison between first and fourth trials, $P<0.001$ at both timescales). However, the extent of cross-habituation to structurally similar test odorants differed between the two paradigms (Table 1; Fig. 3). Mice habituated using the short-timescale paradigm exhibited no cross-habituation to even the most chemically similar odorants in this series (Tukey HSD, $P<0.05$ for all test odorants), reflecting the electrophysiological findings of Wilson (2000b). In contrast, mice habituated at the longer timescale crosshabituated significantly to odorants differing in chain length by up to three carbon atoms (Tukey HSD, $P>0.05$ in all cases except $n$-hexanoic acid), a result consistent with previous data (Cleland et al. 2002; Cleland and Narla 2003). These results demonstrate that the timescale of habituation alone is sufficient to determine the stimulus specificity of olfactory habituation memory.

\section{Different habituation memory mechanisms}

The above measurements of habituation memory persistence and specificity at two experimental timescales indicate that the ap- 
A

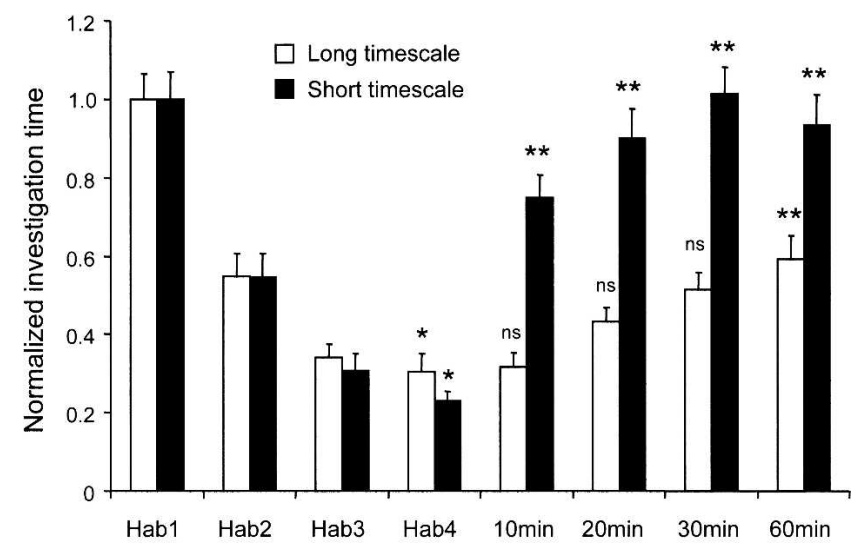

B

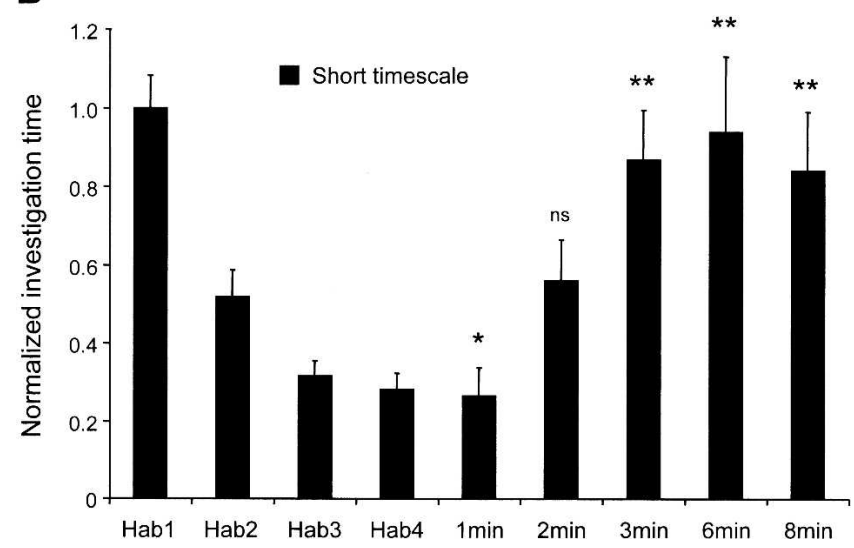

Figure 2. Effect of habituation timescale on memory persistence. Data are presented as investigation times normalized to habituation trial 1 (Hab1). Error bars denote standard error; the criterion for significance is $\alpha=0.05$. $\left({ }^{*}\right)$ Investigation times significantly different from that of Hab1; $\left({ }^{* *}\right)$ investigation times significantly different than that of habituation trial 4 (Hab4); (ns) not significant. (A) When mice were habituated to the bedding odors of a conspecific male using the long-timescale paradigm, the memory for the habituated odor persisted for $>30 \mathrm{~min}$ after the end of trial 4 (open bars). At the shorter timescale, the habituation memory was less persistent; mice did not remember the habituated odor for even 10 min after trial 4 (solid bars). (B) To more accurately determine memory persistence following habituation at the short timescale, mice were habituated to the bedding odors of a conspecific male and tested at latencies of either 1, 2, 4, 6, or 8 min following the end of trial 4. Mice remembered the habituated odor for $\geq 2 \mathrm{~min}$, but $<3 \mathrm{~min}$.

parently contrasting results observed in previous studies can be entirely attributed to the different timescales of the habituation protocols employed. In order to assess whether separate neural mechanisms were responsible for these different habituation responses, we sought to dissociate them pharmacologically.

$\mathrm{N}$-methyl-D-aspartate (NMDA)-type glutamate receptors in the olfactory bulb have been demonstrated to play a role in odor learning (Shimshek et al. 2005; Mandairon et al. 2006b), whereas metabotropic glutamate receptors of the mGluR II/III family are responsible for response adaptation in the anterior piriform cortex (Best and Wilson 2004). We therefore tested whether systemic infusions of the specific NMDA receptor antagonist MK801 or the specific mGluR II/III antagonist LY341495 could affect olfactory habituation at each timescale. Having already demonstrated significant differences between the effects of the short- and long-timescale paradigms, we analyzed the data from the two timescales separately. Analyses of variance indicated that the systemic drug treatments caused significant changes in habituation behavior at both timescales (Short timescale: $F_{\text {trial }(3,224)}=24.79, P<0.001 ; F_{\text {drug }(2,224)}=44.88, P<0.001$; $F_{\text {trial }} \times \operatorname{drug}(6,224)=9.42, P<0.001 ;$ Long timescale: $F_{\text {trial }(3,264)}=33.37, P<0.001 ; F_{\text {drug }(2,264)}=24.69, P<0.001$; $\left.F_{\text {trial } \times \text { drug }(6,264)}=5.49, P<0.001\right)$.

Vehicle-injected control mice habituated normally to repeated presentations of conspecific odor at both the short and long timescales (Tukey HSD, comparison between first and fourth trials, $P<0.001$ in both cases; Fig. 4). Habituation at the short timescale was impaired by the mGluR II/III antagonist LY341495 (Tukey HSD, comparison between first and fourth trials, $P>0.05$ ), but was not affected by blockade of NMDA receptors with MK-801 $(P<0.001)$. In contrast, at the long timescale, mice treated with MK-801 failed to habituate to odorant presentations $(P>0.05)$, whereas mice treated with LY341495 habituated normally $(P<0.001)$. In summary, olfactory habituation memory is sensitive to antagonists of different classes of glutamate receptor depending on the timescale of repeated odorant presentations.

\section{Different structures mediating habituation learning}

Behavioral studies in rats have demonstrated that short-timescale habituation is impaired by mGluR II/III antagonists targeted specifically to the aPC (Best et al. 2005; Yadon and Wilson 2005). We sought in turn to identify the neural structures underlying longtimescale, NMDA-dependent habituation. Specifically, given substantial existing evidence for the importance of NMDA receptors in olfactory bulb plasticity (Wilson et al. 1996; Satou et al. $2005,2006)$, we surgically implanted cannulae into both olfactory bulbs in a group of 12 mice to test the acute behavioral effects of targeted intrabulbar administration of MK-801.

Following bilateral infusions of either MK-801 or saline vehicle into the olfactory bulbs, mice were habituated to conspecific odor as described above using either the short-or longhabituation timescale (Fig. 1). Analysis of variance revealed significant effects of timescale, drug, and trial on investigation behavior $\left(F_{\text {timescale }(1,163)}=170.461, P<0.001 ; F_{\text {drug }(1,163)}=10.306\right.$, $\left.P<0.005 ; F_{\text {trial }(3,163)}=52.569, P<0.001\right)$, and significant interactions between timescale and trial $\left(F_{\text {timescale }} \times \operatorname{trial}(3,163)=3.343\right.$, $P<0.05)$, trial and drug $\left(F_{\text {trial }} \times \operatorname{drug}(3,163)=6.986, P<0.001\right)$, and timescale and drug $\left(F_{\text {timescale }} \times \operatorname{drug}(1,163)=8.347, P<0.005\right)$.

Control mice infused with saline vehicle habituated to the conspecific odor at both the short and long timescales (Tukey HSD, comparison between first and fourth trials, $P<0.001$ in both cases; Fig. 5). Following bilateral infusion of the NMDA receptor antagonist MK-801 into the olfactory bulbs, mice subjected to the short-timescale paradigm habituated normally $(P<0.001)$, whereas mice habituated using the long-timescale paradigm did not habituate $(P>0.05)$. These results demonstrate the involvement of bulbar NMDA receptors in the formation of olfactory habituation memory induced over a long timescale.

\section{Drug administration does not impair odor detection}

All mice subjected to pharmacological studies were tested after these studies were completed to confirm that the administration of either drug at either timescale did not impair their capacity to smell. Following the systemic administration of MK-801, LY341495, or saline vehicle, mice underwent four habituation trials using clean bedding followed by a fifth test trial using conspecific odor (soiled bedding). At the short timescale, under all three drug treatment conditions, we observed no effects attrib- 
Table 1. Dilutions and cross-habituation among straight-chain carboxylic acid odorants

\begin{tabular}{|c|c|c|c|}
\hline Odor $^{a}$ & $\begin{array}{l}\text { Percent } \\
\text { (vol/vol) } \\
\text { dilution }^{\text {b }}\end{array}$ & $\begin{array}{c}\text { Short timescale: } \\
\text { Discriminated from } \\
\text { acetic acid? }\end{array}$ & $\begin{array}{l}\text { Long timescale: } \\
\text { Discriminated from } \\
\text { acetic acid? }\end{array}$ \\
\hline Acetic acid (2) $\mathrm{COOH}$ & 0.0070 & - & - \\
\hline Propanoic acid (3)COOH & 0.0345 & Yes, $P<0.001$ & No, $P>0.05$ \\
\hline$n$-Butanoic acid (4) $\mathrm{COOH}$ & 0.1070 & Yes, $P<0.001$ & No, $P>0.05$ \\
\hline$n$-Pentanoic acid (5)COOH & 1.1635 & Yes, $P<0.005$ & No, $P>0.05$ \\
\hline$n$-Hexanoic acid (6) $\mathrm{COOH}$ & 6.4330 & Yes, $P<0.001$ & Yes, $P<0.001$ \\
\hline
\end{tabular}

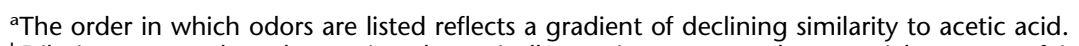

${ }^{b}$ Dilutions were selected to emit a theoretically consistent vapor-phase partial pressure of 1.0 Pa for each odorant when dissolved in mineral oil (see Materials and Methods).

utable to drug administration $\left(F_{\text {trial }(4,340)}=54.318, P<0.001\right.$; $\left.F_{\text {drug }(2,340)}=0.79, P>0.05 ; F_{\text {drug }} \times \operatorname{trial}(8,340)=0.67, P>0.05\right)$; investigation of the conspecific odor was significantly higher than that of the clean bedding after habituation under all three conditions (Tukey HSD, comparison between fourth and fifth trials, $P<0.001$ in all cases; Fig. 6A). At the long timescale, a significant drug effect on investigation times was observed $\left(F_{\text {trial }(4,340)}=95.17, P<0.001 ; F_{\text {drug }(2,340)}=13.29, P<0.001\right.$; $\left.F_{\text {drug }} \times \operatorname{trial}(8,340)=7.19, P<0.001\right)$, likely owing to nonselective reductions in overall investigation times across trials following drug treatments. In concordance with this interpretation, under all three drug conditions, mice investigated the conspecific odor significantly more than they did the clean bedding (Tukey HSD, comparison between fourth and fifth trials, $P<0.001$ in all cases; Fig. 6B). Hence, drug administration did not impair the ability of the mice to detect odor stimuli at either timescale.

We confirmed similarly that the blockade of olfactory bulb NMDA receptors by direct infusion of MK-801 did not impair odor detection. Using the same experimental paradigm, neither saline nor MK-801 infusions disrupted odor detection at either the short timescale $\left(F_{\mathrm{drug}(1,85)}=17.952, P<0.005\right.$; $F_{\text {trial }(4,85)}=17.952, P<0.001 ; F_{\mathrm{drug} \times \operatorname{trial}(4,85)}=1.060, P>0.05$; Tukey HSD, comparison between fourth and fifth trials, $P<0.001$; Fig. 6C) or the long timescale $\left(F_{\operatorname{drug}(1,80)}=2.734\right.$, $P>0.05 ; F_{\text {trial }(4,80)}=40.695, p<0.001 ; F_{\text {drug } \times \operatorname{trial}(4,80)}=3.971$,
$P<0.01$; Tukey HSD, comparison between fourth and fifth trials, $P<0.001$; Fig. 6D).

\section{Discussion}

We describe two qualitatively different mechanisms of olfactory habituation memory in mice, distinguished from one another by persistence, specificity, location, and pharmacological dependence. The first, short-timescale mechanism is observable with a habituation paradigm comparable to the protocol used by Wilson and colleagues (Wilson 1998b; Best and Wilson 2004; Best et al. 2005), utilizing brief (20-sec) odor presentations and short $(10$-sec) intervals between successive presentations. The resulting habituation persists only briefly ( $2 \mathrm{~min})$, is highly specific for the habituation odor, is mediated by the aPC, and is sensitive to blockade of mGluR II/III, but not NMDA, receptors (Best et al. 2005; Yadon and Wilson 2005). In contrast, a different, longtimescale habituation protocol based on that of Linster, Cleland, and colleagues (Cleland et al. 2002; Linster et al. 2002; Mandairon et al. 2006b,c) utilizes longer (50-sec) odor presentations separated by 5 -min intertrial intervals (ITIs). The resulting habituation persists substantially longer (up to $30 \mathrm{~min}$ ), generalizes to structurally and perceptually similar odorants to an extent consistent with prior studies (Cleland et al. 2002), is mediated within the olfactory bulb, and is impaired by the blockade of NMDA receptors but not mGluR II/III receptors. This selective
A

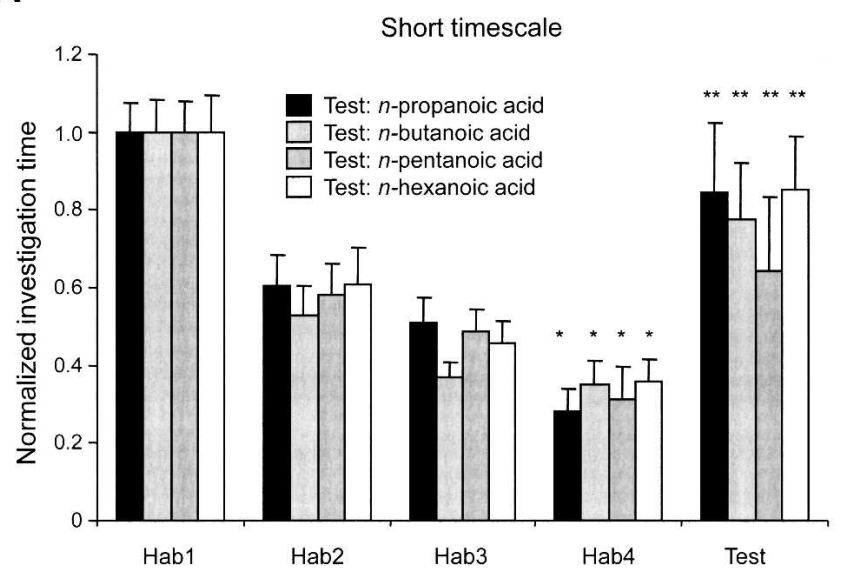

B

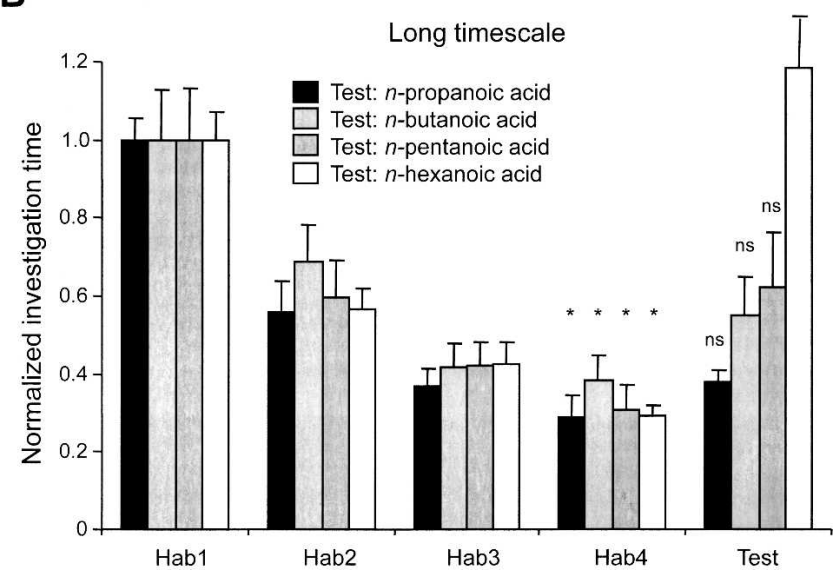

Figure 3. Effect of habituation timescale on memory specificity (cross-habituation to structurally and perceptually similar odorants). Data are presented as investigation times normalized to habituation trial 1 (Hab1). Error bars denote standard error; the criterion for significance is $\alpha=0.05$. (*) Investigation times significantly different from that of Hab1; $\left(^{* *}\right)$ investigation times significantly different than that of habituation trial 4 (Hab4); (ns) not significant. The short-timescale paradigm yields correspondingly shorter absolute investigation times (data not shown). ( $A$ ) Odor memory is highly specific when the timescale of habituation is short. Mice habituated to acetic acid did not cross-habituate to any of the test odorants; i.e., they investigated each test odorant for a significantly longer time than they investigated the habituation odorant $(P<0.05$ in all cases). (B) Mice cross-habituated to similar odorants when habituated at the longer timescale. Mice habituated to acetic acid did not investigate the 3-carbon propanoic acid, the 4-carbon butanoic acid, or the 5-carbon pentanoic acid to a significantly greater degree than they did the habituated odorant $(P>0.05$ in all cases), indicating cross-habituation. Mice did not cross-habituate to the 6-carbon hexanoic acid $(P<0.05)$. 
A

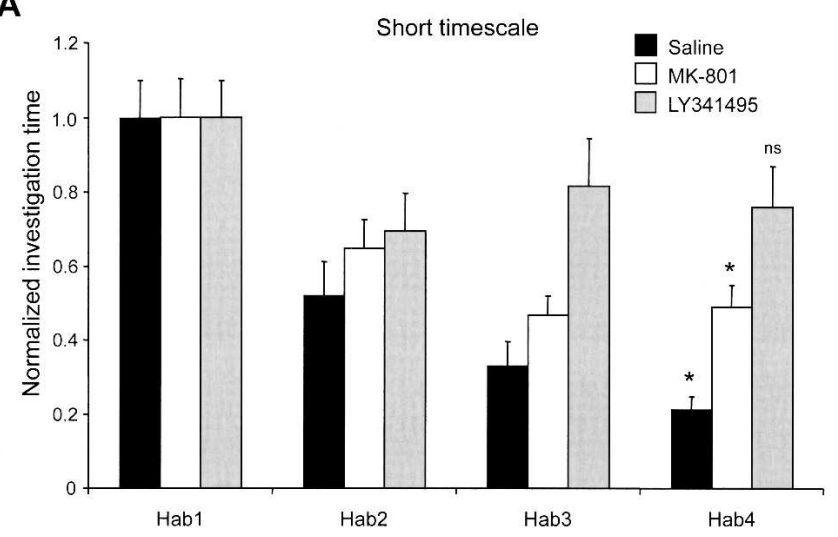

B

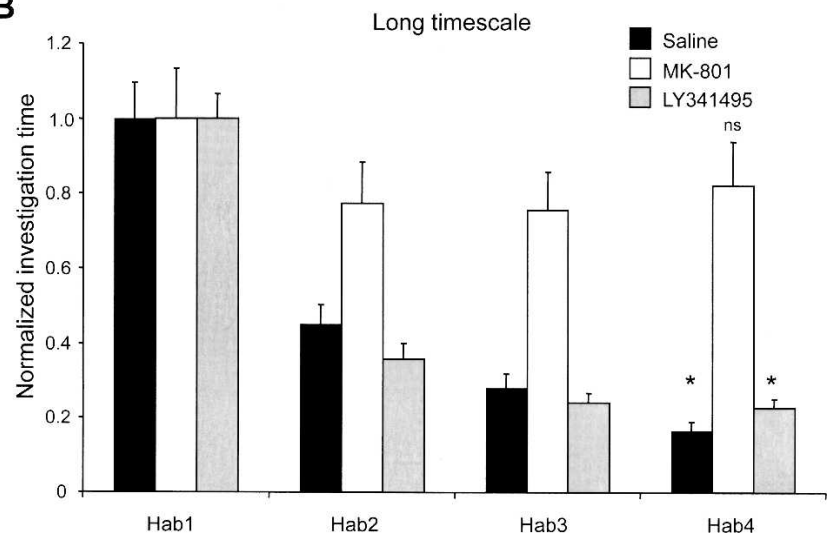

Figure 4. Effect of systemic drug administration on habituation at the two experimental timescales. Data are presented as investigation times normalized to habituation trial 1 (Hab1). Error bars denote standard error; the criterion for significance is $\alpha=0.05$. $\left(^{*}\right)$ Investigation times significantly different from that of Hab1; (ns) not significant. (A) Effect of drug administration on habituation at the short timescale. Saline-injected controls (solid bars) significantly habituated to the odor of a male conspecific over four trials $(P<0.05)$. When injected with the NMDA receptor antagonist MK-801 (open bars), mice still habituated normally to the odor $(P<0.05)$. Injection of the mGluR II/III antagonist LY341495 (shaded bars), however, impaired habituation at this timescale $(P>0.05)$. $(B)$ Effect of drug administration on habituation at the long timescale. Saline-injected controls (solid bars) significantly habituated to the odor of a male conspecific over four trials $(P<0.05)$. Injection of the NMDA receptor antagonist MK-801 (open bars) impaired habituation at this longer timescale $(P>0.05)$. When injected with the mGluR II/III antagonist LY341495 (shaded bars), however, mice still habituated normally to the odor $(P<0.05)$.

impairment of longer-timescale olfactory learning protocols by NMDA is also consistent with earlier odor discrimination studies in which rats' ability to discriminate odors was affected by NMDA receptor blockade at long, but not short, ITIs (Staubli et al. 1989). These results indicate that there are at least two qualitatively distinct neural mechanisms underlying olfactory habituation, and that these mechanisms support habituation memory at two different timescales.

The timescales of the two habituation protocols used in this study differed in both odor presentation and intertrial interval durations in order to replicate the procedures of existing olfactory habituation studies. Both the duration of stimulus sampling (dependent on odor presentation times) and the interval between samples can modulate learning, and it remains unclear whether the multiple effects of timescale reported here may depend more strongly on one or the other of these two variables, or on their interaction. Based on our present results and their correspondence with electrophysiological data from these two neural structures, we propose that odorant exposure elicits two physiological responses relevant to habituation. First, the release of glutamate from odor-activated mitral cells onto aPC pyramidal neurons induces an mGluR II/III-mediated homosynaptic depression of mitral-pyramidal synaptic strengths (Best and Wilson 2004; Linster et al. 2007). This depression underlies response adaptation in pyramidal cells as well as short-term behavioral habituation. These effects can be measured using a short-timescale habituation paradigm; however, when a sufficiently long-timescale paradigm is employed, this synaptic depression and its behavioral effects recover to baseline levels before the presentation of the next stimulus. Second, the same olfactory activation also initiates an NMDA receptor-dependent response in the olfactory bulb, which if reinforced by sufficiently extended stimulation results in a relatively persistent ( $>30$-min) habituation memory therein.

Interpretation of these data in terms of the relative persistence of memory traces also enables direct comparison with clas-
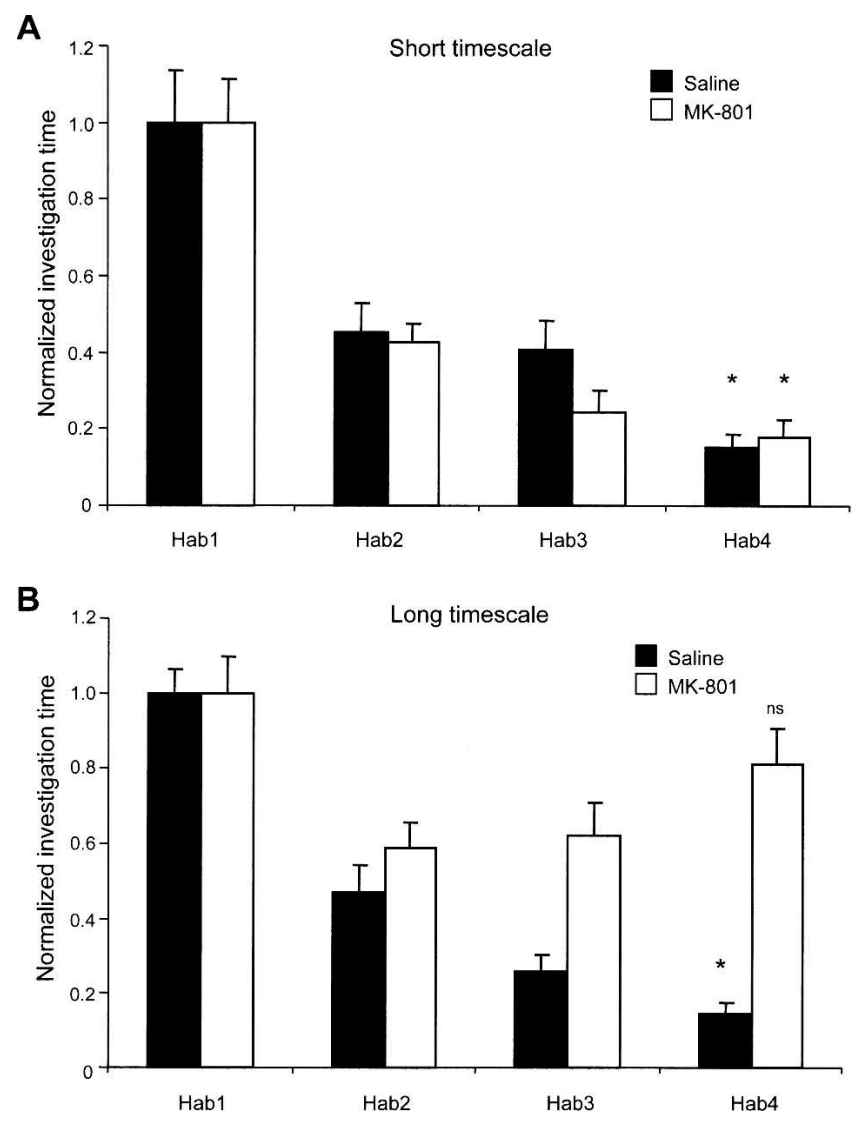

Figure 5. Effect of NMDA receptor blockade in the olfactory bulbs on habituation at the two experimental timescales. Data are presented as investigation times normalized to habituation trial 1 (Hab1). Error bars denote standard error; the criterion for significance is $\alpha=0.05$. $\left(^{*}\right)$ Investigation times significantly different from that of Hab1. (A) Effect of NMDA receptor blockade in the olfactory bulbs at the short timescale. Saline-infused controls (solid bars) significantly habituated to the odor of a male conspecific over four trials $(P<0.05)$. When the NMDA receptor antagonist MK- 801 was infused bilaterally into the olfactory bulbs, mice still habituated normally to the odor (open bars; $P<0.05$ ). (B) Effect of NMDA receptor blockade in the olfactory bulbs at the long timescale. Saline-infused controls (solid bars) significantly habituated to the odor of a male conspecific over four trials $(P<0.05)$. Bilateral infusion of the NMDA receptor antagonist MK-801 into the olfactory bulbs (open bars) impaired habituation at this longer timescale $(P>0.05)$. 
A

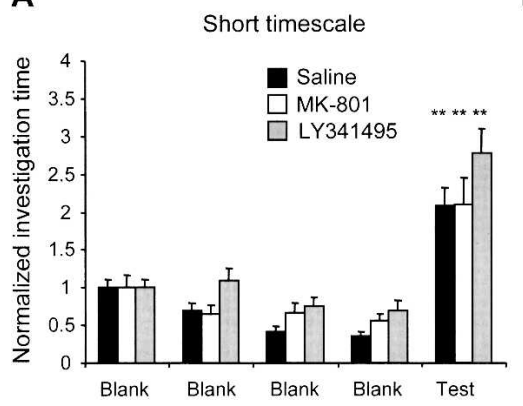

C

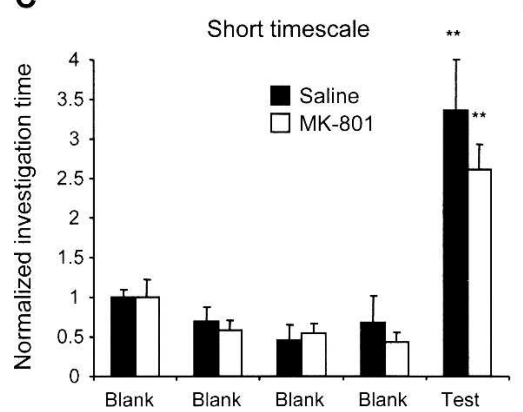

B

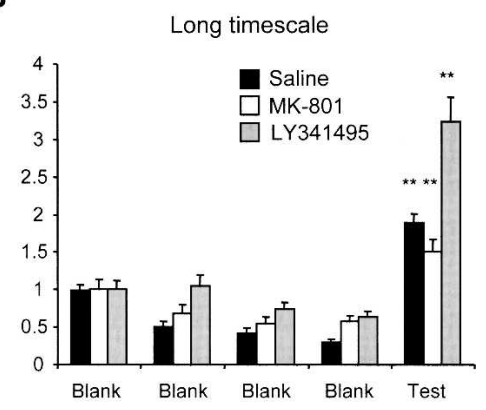

D

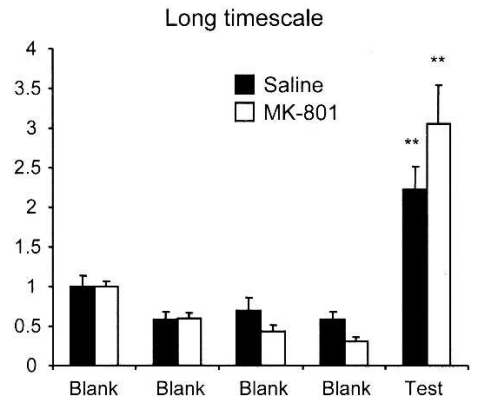

Figure 6. Drug administration does not impair odor detection. Data are presented as investigation times normalized to trial 1. Error bars denote standard error; the criterion for significance is $\alpha=0.05$. $\left.{ }^{(*}\right)$ Investigation times significantly different than that of the fourth blank trial. Fifteen minutes after the intraperitoneal injection $(A, B)$ or bilateral infusion into the olfactory bulbs $(C, D)$ of a test drug or saline vehicle, mice were habituated over four trials to tea balls filled with clean bedding. Conspecific odor (soiled bedding) was then presented during the test trial, eliciting significantly longer investigation times than any of the previous trials irrespective of drug treatments $(P<0.001$ in all cases).

sical and contemporary theories of learning. The decay of habituation with time reflects Ebbinghaus' power law of forgetting, $R=e^{-t / S}$, where $R$ indicates memory retention, $t$ indicates time, and $S$ indicates the "strength" of learning such that stronger memories retard their own rate of decay in time (Ebbinghaus 1885). In this formulation, olfactory habituation as shown here over the longer timescale corresponds with stronger learning than that effected at the shorter timescale (Fig. 2), recapitulating well-established effects of spaced versus massed learning (Jost 1897; Beck and Rankin 1995; Menzel et al. 2001; Rose and Rankin 2001). These phenomena have been incorporated into contemporary real-time learning theories; for example, the SOP model and its derivatives incorporate "nodes" with embedded activity states such that the decay rate $p d 1$ of informational elements from the primary active state $A 1$ into a refractory state $A 2$ corresponds to the rate of short-term habituation (Brandon et al. 2003). While these nodes can be considered as representing individual stimuli, the present results are better modeled by mapping the odor stimulus representation onto two such nodes, independent and with different decay rates $p d 1$, that receive parallel inputs and are linked together at their respective outputs $A 1$ with an intersection filter (Boolean AND) such that behavioral responses to odor presentation will occur only when both nodes are capable of elevating substantial numbers of elements into the active state $A 1$. That is, habituation will be exhibited to the extent that either of the two nodes is refractory. Other SOP-based models of the present results, e.g., serially connected nodes reflecting the neural connectivity of the olfactory bulb and piriform cortex, are also plausible.

The involvement of NMDA receptors in olfactory bulb plasticity has been established in studies of both bulbar cellular activity (Ennis et al. 1998; Galan et al. 2005; Satou et al. 2005, 2006) which can be prevented by blocking olfactory bulb NMDA receptors (Satou et al. 2006). The results of the present study clearly indicate a role for such olfactory bulb NMDA receptor-mediated plasticity in habituation learning at a certain timescale.

In summary, simply varying the timescale of odor stimulus presentation revealed two qualitatively different neural mechanisms underlying habituation learning in mice. Odorants were presented at two timescales selected for ready comparison with existing studies exhibiting contrasting results. The habituation memories formed at these two presentation timescales differed in their persistence and specificity, were mediated by different neural structures, and were sensitive to different pharmacological antagonists.

\section{Materials and Methods}

\section{Subjects}

A total of 58 male CD-1 mice (Charles River), aged 2 mo at the beginning of the study, served as subjects. Thirty-one of these mice (Group 1A of 24 and Group 1B of seven) were used for memory persistence and pharmacology testing. A separate cohort of 15 mice (Group 2) was used for specificity measurements, and a third cohort of 12 mice (Group 3) was used in cannulation studies. Mice were housed in groups of 3-4 in standard laboratory cages and kept on a reversed 12:12 light cycle. Food and water were available ad libitum. All experiments were carried out under a protocol approved by the Cornell University Institutional Animal Care and Use Committee in accordance with NIH guidelines.

\section{Odors}

Six male CD-1 mice, $\sim 6$ mo old at the start of the experiment and that had been housed individually for at least $1 \mathrm{mo}$, served as odor donors. Individually housed males have been reported to 
produce the same distinctive chemicals in their urine as known dominant males (Jones and Nowell 1975; Wilson 2000b). Soiled bedding ( $\sim 3.5 \mathrm{~g})$ from one of the donor mouse cages was placed in a tea ball for presentation to subject mice (conspecific odor).

For measurements of the specificity of habituation memory (see Behavioral Testing), we used a homologous series of straightchain carboxylic acids including acetic acid, propanoic acid, $n$-butanoic acid, $n$-pentanoic acid, and $n$-hexanoic acid (SigmaAldrich). All odors were diluted with mineral oil to achieve a theoretical vapor-phase partial pressure of $1.0 \mathrm{~Pa}$ (Cleland et al. 2002; Wiltrout et al. 2003); corresponding volume/volume dilutions are listed in Table 1 . For odor presentations, $60-\mu \mathrm{L}$ aliquots of diluted odorant or plain mineral oil were deposited onto filter paper and then placed inside a tea ball.

\section{Behavioral testing}

Mice were tested at $1100 \mathrm{~h}, 2 \mathrm{~h}$ into their dark cycle. For testing, mice were separated into individual plastic cage boxes without bedding or food; microisolator cage lids were used to minimize environmental odors entering the boxes during testing. Two habituation paradigms were used that differed only in timescale (i.e., the duration and frequency of odorant stimulus presentations; Fig. 1). The two timescales were selected to match, as closely as practically possible, the odor presentation and ITI durations used in existing olfactory habituation studies exhibiting conflicting results (short timescale: Wilson 1998a; Best and Wilson 2004; Best et al. 2005; long timescale: Cleland et al. 2002; Linster et al. 2002; Wiltrout et al. 2003; Mandairon et al. 2006b,c).

\section{Persistence}

Thirty-one mice (Group 1) were used to measure the persistence of the memory for the habituated odor using both long- and short-timescale habituation paradigms. Long-timescale habituation consisted of five stimulus presentations, each lasting $50 \mathrm{sec}$, with 5-min ITIs. The first presentation consisted of clean bedding to habituate the mice to the non-olfactory novelty such as the presence of the tea ball (Fig. 1, blank); this was followed by four presentations of soiled bedding sourced from a single male conspecific (conspecific odor). Investigation time, defined as the amount of time a mouse spent actively sniffing with its nose $\leq 1$ $\mathrm{cm}$ from the tea ball, was recorded with a stopwatch. Habituation, a progressive reduction in investigation time over successive trials, indicates that the animal remembers its prior experience with the stimulus and no longer investigates it as if it were novel. Short-timescale habituation trials consisted of the same five stimulus presentations, except that presentations were only $20 \mathrm{sec}$ long with 10-sec ITIs. The ITI used corresponds to that used in prior short-timescale habituation studies (Wilson 1998a,b; Best and Wilson 2004). The duration of odor presentations was modestly increased in comparison with these studies so as to accommodate the experimental design; however, the actual investigation times demonstrated by the mice in this study are on the order of the 4-sec odor exposure time used in the relevant habituation studies (Best and Wilson 2004).

Following the end of the last habituation trial, the same (habituated) odor was presented again after 10-, 20-, 30-, and 60-min latencies. Investigation times that did not significantly differ from that of the last habituation trial indicated that the mouse remembered the habituated odor. This straightforward method is likely to overestimate the absolute duration of habituation memory owing to the refresher effect of earlier test trials, but does not prevent progressive recovery from habituation and enables ready comparison of the two different timescales.

Mice were then tested at shorter latencies $(1,2,3,4,6$, and $8 \mathrm{~min}$ ) after the last habituation trial using only the shorttimescale habituation protocol. Because the intervals between the last habituation trial and the test trial were very short, each mouse was tested on only a single latency on any given day. Odors from different donor mice were used on each day of testing to avoid any long-term cumulative effects.

\section{Specificity}

Fifteen CD-1 mice (Group 2) were used to measure the specificity of habituation at each timescale. Mice were tested using both long- and short-timescale habituation paradigms as described above. However, unscented mineral oil was presented for the first (blank) trial in lieu of fresh bedding, whereas acetic acid (a twocarbon carboxylic acid odorant diluted in mineral oil) was presented for the four subsequent habituation trials. Subsequent test trials measured the degree of cross-habituation from the habituated odorant to each novel test odorant. Specifically, two test trials, each consisting of a novel 3-, 4-, 5-, or 6-carbon straightchain carboxylic acid odorant diluted in mineral oil (Table 1; see Odors section, above), were subsequently performed after each habituation series at latencies equal to the habituation ITI. All four test odorants were thereby presented over $2 \mathrm{~d}$ according to a counterbalanced schedule. Consequently, each mouse was habituated twice to acetic acid. Habituation series were separated by at least $24 \mathrm{~h}$ to minimize cumulative effects. We confirmed this with statistical analyses; whereas there was a modest effect of day on investigation times at both timescales $\left(F_{\text {long }(1,84)}=5.538\right.$; $\left.P<0.05 ; F_{\text {short (1.96) }}=6.746 ; P<0.05\right)$, in neither case was there a significant effect of day on investigation during the fourth habituation trial $(P>0.05$ at both timescales), indicating that all test trials were performed from the same baseline habituation level. Furthermore, the order of test odorant presentation was counterbalanced in order to prevent any accumulated effects from systematically influencing spontaneous discrimination results.

\section{Pharmacology}

Twenty-four CD-1 mice (Group 1A) previously used to test memory persistence were then used in pharmacological studies to help ascertain underlying neural mechanisms. Pharmacological experiments followed the same protocol and employed the same odorants as persistence experiments, except that the test trial was always performed at a latency equal to the habituation ITI, and drugs were administered intraperitoneally $15 \mathrm{~min}$ before the trials began.

Two specific antagonists targeting different glutamate receptors were used. First, MK-801 ([+]-5-methyl-10,11, dihydro-5Hdibenzo[a,d]cyclohepten-5,10-imine maleate; Sigma-Aldrich; 0.2 $\mathrm{mg} / \mathrm{kg}$ IP) was used to block NMDA receptors known to exist in the olfactory system (Wilson et al. 1996). Second, LY341495 (Tocris Bioscience; $2.5 \mathrm{mg} / \mathrm{kg} \mathrm{IP}$ ), a metabotropic glutamate receptor II/III antagonist capable of crossing the blood-brain barrier (O'Neill et al. 2003; Rasmussen et al. 2004), was used to block the activity of mGluR II/III metabotropic glutamate receptors in the olfactory system (Best and Wilson 2004).

\section{Local olfactory bulb drug infusions}

Twelve mice (Group 3) were anesthetized with a mixture of ketamine $(80 \mathrm{mg} / \mathrm{kg})$ and xylazine $(6 \mathrm{mg} / \mathrm{kg})$ intraperitoneally and placed in a stereotaxic instrument (Kopf Instruments). Double guide cannulae (26-gauge; Plastics One, Inc.) were implanted such that both olfactory bulbs were cannulated for drug infusions at the following coordinates relative to bregma: $+5.0 \mathrm{~mm}$ anteroposterior, $\pm 0.5 \mathrm{~mm}$ mediolateral, and $-1.5 \mathrm{~mm}$ dorsoventral. Implants were secured in place with screws and dental cement, and dummy cannulae were placed inside the guides to protect against blockage and infection. Mice were allowed $14 \mathrm{~d}$ for recovery prior to behavioral testing.

For local drug infusions before behavioral testing, mice were briefly anesthetized with $2 \%$ isoflurane gas in oxygen. One of two solutions was infused bilaterally into the olfactory bulbs: $0.9 \%$ sterile saline vehicle (Baxter Healthcare Corporation) or 0.2 mM MK-801 ([+]-5-methyl-10,11, dihydro-5H-dibenzo[a,d]cyclohepten-5,10-imine maleate; Sigma-Aldrich) in vehicle. Infusion cannulae protruded $1 \mathrm{~mm}$ beyond the guide cannulae. A total volume of $2 \mu \mathrm{L}$ of each solution was administered into each olfactory bulb at a rate of $2 \mu \mathrm{L}$ per minute. Infusion cannulae were left in place for $1 \mathrm{~min}$ after the infusion. These infusion 
volumes were determined by injecting $2 \%$ pontamine blue dye, 2 $\mu \mathrm{L}$ of which diffused adequately through each olfactory bulb without invading other cortical areas.

\section{Odor detection test}

After habituation testing, all mice used in pharmacological studies (Groups 1 and 3) were tested to ensure that they could adequately smell the test odorants used under every drug treatment condition. Following the same basic protocol, and under both short- and long-timescale protocols, mice were presented with clean bedding four times, to which they habituated, and then were presented soiled bedding (conspecific odor) in a test trial. A significant increase in investigation time during the test trial indicated that the drugs did not impair olfactory perception. This procedure was followed for both systemic injections and local olfactory bulb infusions.

\section{Data analysis}

All data were recorded as investigation time in seconds. Data were plotted using Microsoft Excel (Microsoft Corporation) and are presented as mean \pm standard error. Outlier trials exhibiting investigation times of more than two standard deviations from the mean for a given trial were excluded from analysis. In our experience, these outlier trials occur when animals are distracted or frightened by external stimuli. Statistical analyses were performed with SPSS 14.0 (SPSS Inc.). Analyses of variance were performed on all data sets, followed by post hoc testing using Tukey's honestly significant difference criterion $(\alpha=0.05)$ to assess the differences between individual trials.

\section{Acknowledgments}

Funding for this project was provided by a departmental Student Research Grant in Animal Behavior and an AAUW American Fellowship to A.M.M. and NSF award IOB 0388981 to C.L. and D.A.W.

\section{References}

Beck, C.D. and Rankin, C.H. 1995. Long-term habituation is produced by distributed training at long ISIs and not by massed training or short ISIs in Caenorhabditis elegans. Anim. Learn. Behav. 25: 446-457.

Best, A.R. and Wilson, D.A. 2004. Coordinate synaptic mechanisms contributing to olfactory cortical adaptation. J. Neurosci. 24: $652-660$.

Best, A.R., Thompson, J.V., Fletcher, M.L., and Wilson, D.A. 2005. Cortical metabotropic glutamate receptors contribute to habituation of a simple odor-evoked behavior. J. Neurosci. 25: 2513-2517.

Brandon, S.E., Vogel, E.H., and Wagner, A.R. 2003. Stimulus representation in SOP: I. Theoretical rationalization and some implications. Behav. Processes 62: 5-25.

Brennan, P.A. and Keverne, E.B. 1997. Neural mechanisms of mammalian olfactory learning. Prog. Neurobiol. 51: 457-481.

Brennan, P.A., Schellinck, H.M., de la Riva, C., Kendrick, K.M., and Keverne, E.B. 1998. Changes in neurotransmitter release in the main olfactory bulb following an olfactory conditioning procedure in mice. Neuroscience 87: 583-590.

Cleland, T.A. and Narla, V.A. 2003. Intensity modulation of olfactory acuity. Behav. Neurosci. 117: 1434-1440.

Cleland, T.A., Morse, A., Yue, E.L., and Linster, C. 2002. Behavioral models of odor similarity. Behav. Neurosci. 116: 222-231.

Ebbinghaus, H. 1885. Memory: A contribution to experimental psychology (Ueber das Gedaechtnis). K. Buehler, Leipzig, Germany.

Ennis, M., Linster, C., Aroniadou-Anderjaska, V., Ciombor, K., and Shipley, M.T. 1998. Glutamate and synaptic plasticity at mammalian primary olfactory synapses. Ann. N. Y. Acad. Sci. 855: 457-466.

Fletcher, M.L. and Wilson, D.A. 2002. Experience modifies olfactory acuity: Acetylcholine-dependent learning decreases behavioral generalization between similar odorants. J. Neurosci. 22: RC201. http://www.jneurosci.org/cgi/content/full/22/2/RC201.
Fletcher, M.L. and Wilson, D.A. 2003. Olfactory bulb mitral-tufted cell plasticity: Odorant-specific tuning reflects previous odorant exposure. J. Neurosci. 23: 6946-6955.

Galan, R.F., Weidert, M., Menzel, R., Herz, A.V.M., and Galizia, C.G. 2005. Sensory memory for odors is encoded in spontaneous correlated activity between olfactory glomeruli. Neural Comput. 18: $10-25$.

Halabisky, B., Friedman, D., Radojicic, M., and Strowbridge, B.W. 2000. Calcium influx through NMDA receptors directly evokes GABA release in olfactory bulb granule cells. J. Neurosci. 20: $5124-5134$.

Jones, R.B. and Nowell, N.W. 1975. Effects of clean and soiled sawdust substrates and of different urine types upon aggressive behavior in male mice. Aggress. Behav. 1: 111-121.

Jost, A. 1897. Association strength as a function of the distribution of repetitions (Die Assoziationsfestigkeit in ihrer Abhaengigkeit von der Verteilung der Wiederholungen). Z. Psychol. Physiol. der Sinnesorg. 14: $436-472$.

Kendrick, K.M., Levy, F., and Keverne, E.B. 1992. Changes in the sensory processing of olfactory signals induced by birth in sleep. Science 256: 833-836.

Linster, C., Johnson, B.A., Morse, A., Yue, E., and Leon, M. 2002. Spontaneous versus reinforced olfactory discriminations. J. Neurosci. 22: 6842-6845.

Linster, C., Henry, L., Kadohisa, M., and Wilson, D.A. 2007. Synaptic adaptation and odor-background segmentation. Neurobiol. Learn. Mem. 87: 352-360.

Mandairon, N., Sacquet, J., Garcia, S., Ravel, N., Jourdan, F., and Didier, A. 2006a. Neurogenic correlates of an olfactory discrimination task in the adult olfactory bulb. Eur. J. Neurosci. 24: 3578-3588.

Mandairon, N., Stack, C., Kiselycznyk, C., and Linster, C. 2006b. Broad activation of the olfactory bulb produces long-lasting changes in odor perception. Proc. Natl. Acad. Sci. 103: 13543-13548.

Mandairon, N., Stack, C., and Linster, C. 2006c. Olfactory enrichment improves the recognition of individual components in mixtures. Physiol. Behav. 89: 379-384.

Menzel, R., Manz, G., and Greggers, U. 2001. Massed and spaced learning in honeybees: The role of CS, US, the intertrial interval, and the test interval. Learn. Mem. 8: 198-208.

O’Neill, M.F., Heron-Maxwell, C., Conway, M.W., Monn, J.A., and Ornstein, P. 2003. Group II metabotropic glutamate receptor antagonists LY341495 and LY366457 increase locomotor activity in mice. Neuropharmacology 45: 565-574.

Rasmussen, K., Hsu, M.A., and Vandergriff, J. 2004. The selective mGlu2/3 receptor antagonist LY341495 exacerbates behavioral signs of morphine withdrawal and morphine-withdrawal-induced activation of locus coeruleus neurons. Neuropharmacology 46: 620-628.

Rochefort, C., Gheusi, G., Vincent, J.D., and Lledo, P.M. 2002. Enriched odor exposure increases the number of newborn neurons in the adult olfactory bulb and improves odor memory. J. Neurosci. 22: 2679-2689.

Rose, J.K. and Rankin, C.H. 2001. Analyses of habituation in Caenorhabditis elegans. Learn. Mem. 8: 63-69.

Salin, P.A., Lledo, P.M., Vincent, J.D., and Charpak, S. 2001. Dendritic glutamate autoreceptors modulate signal processing in rat mitral cells. J. Neurophysiol. 85: 1275-1282.

Satou, M., Anzai, S., and Huruno, M. 2005. Long-term potentiation and olfactory memory formation in the carp (Cyprinus carpio L.) olfactory bulb. J. Comp. Physiol. A Neuroethol. Sens. Neural Behav. Physiol. 191: 421-434.

Satou, M., Hoshikawa, R., Sato, Y., and Okawa, K. 2006. An in vitro study of long-term potentiation in the carp (Cyprinus carpio L.) olfactory bulb. J. Comp. Physiol. A Neuroethol. Sens. Neural Behav. Physiol. 192: 135-150.

Schoppa, N.E., Kinzie, J.M., Sahara, Y., Segerson, T.P., and Westbrook, G.L. 1998. Dendrodendritic inhibition in the olfactory bulb is driven by NMDA receptors. J. Neurosci. 18: 6790-6802.

Shimshek, D.R., Bus, T., Kim, J., Mihaljevic, A., Mack, V., Seeburg, P.H., Sprengel, R., and Schaefer, A.T. 2005. Enhanced odor discrimination and impaired olfactory memory by spatially controlled switch of AMPA receptors. PLoS Biol. 3: e354. doi: 10.1371/journal.pbio. 0030354.

Staubli, U., Thibault, O., DiLorenzo, M., and Lynch, G. 1989. Antagonism of NMDA receptors impairs acquisition but not retention of olfactory memory. Behav. Neurosci. 103: 54-60.

Thompson, R.F. and Spencer, W.A. 1966. Habituation: A model phenomenon for the study of neuronal substrates of behavior. Psychol. Rev. 73: 16-43.

Wilson, D.A. 1998a. Habituation of odor responses in the rat anterior piriform cortex. J. Neurophysiol. 79: 1425-1440.

Wilson, D.A. 1998b. Synaptic correlates of odor habituation in the rat anterior piriform cortex. J. Neurophysiol. 80: 998-1001. 
Wilson, D.A. 2000a. Comparison of odor receptive field plasticity in the rat olfactory bulb and anterior piriform cortex. J. Neurophysiol.

84: $3036-3042$.

Wilson, D.A. 2000b. Odor specificity of habituation in the rat anterior piriform cortex. J. Neurophysiol. 83: 139-145.

Wilson, D.A. and Leon, M. 1988. Spatial patterns of olfactory bulb single-unit responses to learned olfactory cues in young rats. $J$. Neurophysiol. 59: 1770-1782.

Wilson, D.A., Sullivan, R.M., Gall, C.M., and Guthrie, K.M. 1996. NMDA-receptor modulation of lateral inhibition and c-fos expression in olfactory bulb. Brain Res. 719: 62-71.
Wiltrout, C., Dogra, S., and Linster, C. 2003. Configurational and nonconfigurational interactions between odorants in binary mixtures. Behav. Neurosci. 117: 236-245.

Yadon, C.A. and Wilson, D.A. 2005. The role of metabotropic glutamate receptors and cortical adaptation in habituation of odor-guided behavior. Learn. Mem. 12: 601-605.

Received September 27, 2007; accepted in revised form January 8, 2008. 


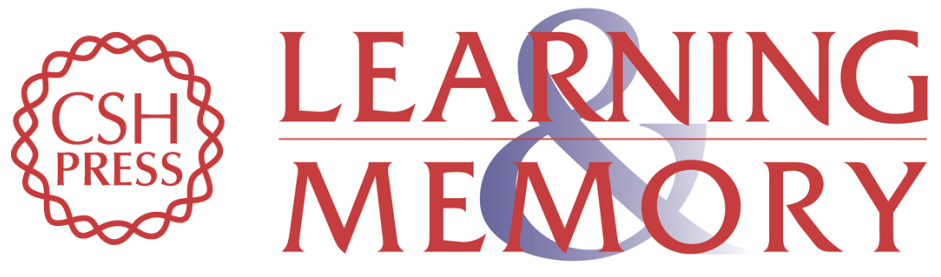

\section{Distinct neural mechanisms mediate olfactory memory formation at different timescales}

Ann Marie McNamara, Phillip D. Magidson, Christiane Linster, et al.

Learn. Mem. 2008, 15:

Access the most recent version at doi:10.1101//m.785608

References This article cites 41 articles, 13 of which can be accessed free at:

http://learnmem.cshlp.org/content/15/3/117.full.html\#ref-list-1

License

Email Alerting Receive free email alerts when new articles cite this article - sign up in the box at the Service top right corner of the article or click here. 